\title{
World-Views in the SIunits Package
}

\author{
Mark Fischler * \\ Fermi National AcCelerator Laboratory
}

April 13, 2000

And these shall be the measures thereof.

- Ezekiel $48: 16$

\section{Contents}

1 Introduction

1

2 Defining a World View 2

3 Information Needed to Support Each World View 3

4 Deriving the Information From the Defining Statements 4

5 Examples from the Models 5

6 Where is this Computed or Embedded in SIunits 11

\section{Introduction}

The SIunits package allows for the use of world views other than the "standard" (std) view of dimensional quantities length, time, mass, current, temperature, and so forth. A commonly used world view says "we work in units where $c=1$," for example. The package must do the work to support that new "relativistic" world view.

In this note, I want to provide concrete answers to several questions:

- What set of statements defines a world view?

- What does the SIunits Package need to know to be able to support each world view?

- How can this information be derived from the defining statements, in the general case?

- Where are these computations and derivations done in the SIunits package?

As a starting point, let me describe the std world view as having $N_{d}$ dimensioned units labeled $f_{\alpha}$. Thus $f_{1}$ is a meter, $f_{2}$ a kilogram, $f_{3}$ a second, and so forth. $N_{d}$ is seven but that is moot; were we to eliminate Luminosity and/or Amount of Substance, for instance, the arguments in this note would remain unchanged.

\footnotetext{
${ }^{*}$ Email: mf@fnal.gov
} 


\section{Defining a World View}

A world view works with dimensioned units $\delta_{\alpha}$. The view is defined by statements of 3 sorts:

1. Physical constant constraints, of the form

$$
C_{i}=c_{i} \prod_{\alpha=1}^{N_{d}} f_{\alpha}^{C_{i \alpha}}=1 \cdot \prod_{\alpha=1}^{N_{d}} \delta_{\alpha}^{C_{i \alpha}}
$$

For example, in the relativistic model we say that $c=1$. In general one can have $N_{c}$ such constraints, labelled by the index $i . C_{i \alpha}$ is thus a matrix having $N_{c}$ rows corresponding to the $N_{c}$ constraints (the $i$ index), and $N_{d}$ columns corresponding to the fundamental dimensions (the $\alpha$ index).

2. Supression of dimensions: For each physical constant contraint, we choose one of the dimensions which appears in that constraint to a non-zero power, and say that that dimension $\delta_{\alpha}$ is suppressed. That is, because the constraint allows you to express one $\delta_{\alpha}$ in terms of others, we are free to choose one $\delta_{\alpha}$ and require that that dimension never be used when expressing quantities. For example, in the relativistic world view, the sole constraint is that $c=1$, where $c$ is a combination of length and time. This lets us state that the length-like dimension is not used in this world view. Instead, length is expressed in terms of the time-like dimension (and a unit of length would be interpreted in "light-seconds").

3. Denominating quantities. These are a set of $\left(N_{d}-N_{c}\right)$ dimensioned quantities $Q_{\beta}$. They must be chosen in such a way that every unit $f_{\alpha}$ can be expressed as

$$
f_{\alpha}=s_{\alpha} \prod_{\beta} Q_{\beta}^{R_{\alpha \beta}} \prod_{i=1}^{N_{c}} C_{i}^{P_{\alpha \beta}}
$$

That looks a lot more complicated than it is: The meaning is that there has to be some way to get to each $f_{\alpha}$ by multiplying some of the $\left\{Q_{\beta}\right\}$ and some of the physical constraint constants. For example, in the rel model, we choose for the $\left\{Q_{\beta}\right\}$ seconds, Amps, Kelvins, and eVs. Of these, $\mathrm{eV}$ is non-trivial: We can use $\mathrm{eV}$ because a mass (in $\mathrm{kg}$ ) can be expressed in $\mathrm{eV} / \mathrm{c}^{2}$ and $c$ is one of the physical constraint constants of the model.

We can write each dimensioned quantitity $Q_{\beta}$ as

$$
Q_{\beta}=q_{\beta} \prod_{\alpha=1}^{N_{d}} f_{\alpha}^{Q_{\beta \alpha}}
$$

In general, as long as you selected the proper number of denomination quantities, and the dimensions of the constraints and these quantities have no linear dependencies, this set of quantities can be used to denominate the units in the new model. The non-trivial matter of determining the $s_{\alpha}$ and $P$ and $R$ is discussed below.

In principle, we allow for the units in our new model to comprise arbitrary combinations of fundamental dimensions. That is, froming each of the $f_{\alpha}$ can require multiple $Q_{\beta}$. In practice, we mainly stick with one $Q_{\beta}$ leading to each fundemental dimension.

Also, in principle when you go from the $Q_{\beta}$ to the $f_{\alpha}$ you could impose a scale factor. If $Q_{\beta}$ were used only in one $f_{\alpha}$ this factor could be absorbed in $Q_{\beta}$; but we have cases where a $Q_{\beta}$ is used to denominate several dimensions. Nonetheless, we restrict our models to use only factors that can be absorbed into the $Q_{\beta}$. 


\subsection{Numbers of Statements}

A complete world view definition based on $N_{c}$ physical constant constraints will have $N_{c}$ supressed dimensions. Corresponding to each non-supressed dimension we must have one $Q_{\beta}$.

These $N_{c}$ numerical relations and $N_{d}-N_{c}$ choices of dimensioned quantities, plus the $N_{c}$ discrete choices of which dimensions to supress, fully define the world view.

\subsection{Consistency}

The set of physical constant constraints must be consistent, in the sense that the set of $N_{d}$-vectors comprising the powers of each dimension in the various of each constraints must be linearly independant. If this were not the case, then either one or more constraints would be derivable from the others, or the set of constraints can never be satisfied.

One also has to worry about consistency or redundancy in the choice of $Q_{\beta}$. In practice, these are chosen such that each is connected in an obvious way to one of the non-supressed dimensions; the expression of the supressed dimensions is then always possible.

\section{Information Needed to Support Each World View}

There are two pieces of information needed for the SIunits mechanisms to operate:

- A mapping between dimensions $\delta_{\alpha}$ in the new world view and products of powers of fundamental dimensions $f_{\alpha}$ in the std view. This is expressed as lines like $d_{1}=p_{1}+p_{3}$

$$
d_{1}=p_{1}+p_{3}
$$

meaning that if a quantity $Q$ contains $p_{1}$ powers of $f_{1}$ and $p_{3}$ powers of $f_{3}$ then in the new world view it will have $d_{1}$ powers of $\delta_{1}$. In general, this mapping looks like

$$
d_{\beta}=\sum_{\alpha} m_{\beta \alpha} p_{\alpha}
$$

where $\beta$ runs over the non-supressed dimensions in the new model.

For supressed dimensions, $d_{\alpha}=0$.

- For every fundamental dimension $f_{\alpha}$, a scale factor (in the calibration and wv.h files, this is called def_m, def_kg, and so forth). Since this is associated with the fundamental dimensions in the std world view, the new world view needs to know all $N_{d}$ of these even if it has several supressed dimensions itself.

The meaning of these pieces of information is that a given std fundamental quantity (for example, a meter) is expressed in the new view as that scale factor, times a unit with dimensions given those $d_{\alpha}$ which would be non-zero when that $p_{\alpha}$ is one. (Given that we can express any fundamental dimension in the new model, it is then trivial to express any general dimensioned quantity by multiplying the appropriate scales and dimensions.)

For example, in the rel model, def_m $=3.335 \mathrm{E}-09$, and $d_{3}=p_{1}+p_{3}$, and $p_{1}$ appears in no other $d_{\alpha}$. So one meter will be $3.335 \mathrm{E}-09$ units of length (in this model, the unit of length is the light-second).

In terms of equation (2), we can identify $s_{\alpha}$ with the scale factor for $f_{\alpha}$, and the $R_{\alpha \beta}$ $R_{\alpha i}$ and can be used to determine the mapping of equation (4). 


\section{Deriving the Information From the Defining State- ments}

\subsection{Solving for $\mathrm{R}$ and $\mathrm{P}$}

We know the details of the denominating quatities and constraints: Matrices $Q_{\beta \alpha}$ and $C_{i \alpha}$, along with the associated scalar constants. But although we know (or at least take it on faith) that for our choice of constraints and denominating quantities there are matrices $R_{\alpha \beta}$ and $P_{\alpha i}$ that will make it possible for equation (2) to hold, we are not given the values of $R_{\alpha \beta}$ and $P_{\alpha i}$. These need to be derived.

We will start from a horrendous-looking relation and demonstrate how it can be seen as an easily managed matrix equation.

When we insert the definitions of $Q_{\beta}$ (equation (3)) and $C_{i}$ (equation (1)) into equation (2) we get that for each value of $\alpha$,

$$
f_{\alpha}=s_{\alpha} \prod_{\beta}\left(q_{\beta} \prod_{\alpha \prime} f_{\alpha \prime}^{Q_{\beta \alpha \prime}}\right)^{R_{\alpha \beta}} \prod_{i=1}^{N_{c}}\left(c_{i} \prod_{\alpha \prime \prime} f_{\alpha \prime \prime}^{C_{i \alpha \prime \prime}}\right)^{P_{\alpha i}}
$$

where as usual $\beta$ ranges over the denominating quantities.

In this horrendous equation, we first pull out all the scalar-valued terms:

$$
f_{\alpha}=s_{\alpha} \prod_{\beta \prime} q_{\beta}^{R_{\alpha \beta \prime}} \prod_{i \prime=1}^{N_{c}} c_{i \prime}^{P_{\alpha i \prime}} \prod_{\beta} \prod_{i=1}^{N_{c}} \prod_{\alpha \prime} \prod_{\alpha \prime \prime}\left(f_{\alpha \prime}^{Q_{\beta \alpha}}\right)^{R_{\alpha \beta}}\left(f_{\alpha \prime \prime}^{C_{i \alpha}}\right)^{P_{\alpha i}}
$$

Now we temporarily ignore the scalar out front, and do power counting to match powers of each $f_{\alpha \prime}$ in the equation for each $f_{\alpha}$. The products become sums, and on the left hand side we require that only one power of $f_{\alpha}$ appear. So we get the equation

$$
\delta_{\alpha \alpha \prime}=\sum_{\beta} R_{\alpha \beta} Q_{\beta \alpha \prime}+\sum_{i=1}^{N_{c}} P_{\alpha i} Q_{i \alpha \prime}
$$

or in matrix notation,

$$
\mathbf{1}=R Q+P C
$$

Finally, if we form a $N_{d}$ by $N_{d}$ matrix $U$ by using the rows of $Q$ where there are nonsupressed dimensions and rows of $C$ in the gaps, and a matrix $K$ by putting the columns of $R$ where there are non-supressed dimensions and columns of $P$ in the gaps, we find

$$
U K=\mathbf{1}
$$

So, solving for $R_{\alpha \beta}$ and $P_{\alpha i}$ is straightforward: We form $U$ out of $Q$ and $P$, invert to find $K$, and we then read $R$ off the $\left(N_{d}-N_{c}\right)$ columns of $K$ corresponding to non-supressed dimensions, and $P$ off the remaining columns.

\subsection{The Dimension Mapping}

To get the mapping needed (equation (4)) we identify $m_{\beta \alpha}$ with $R_{\alpha \beta}$. That is, we simple read off the coefficients of $\{\mathrm{p} 1 \ldots \mathrm{p} 7\}$ for each non-supressed $\mathrm{d}$ from a column of $R$.

This represents a somewhat arbitrary choice, of associating each non-supressed $\delta_{\alpha}$ with s specific $Q_{\beta}$. Given the definition of $Q$ in equation (3), we can see that if we ignore the $C$ terms, each power of $f_{\alpha}$ that goes into a $Q_{\beta}$ is reflected by the corresponding element $R_{\alpha \beta}$. And it is obvious that the $C$ terms contribute nothing in the mapping: For instance, when the speed of light is used to relate length to time measurements, a factor of $c$ will contribute one power of length and negative one power of time; since $c$ enables you to trade a time for a length, the presence of $c^{n}$ in some $Q$ is a wash in terms of powers of length and time. 


\subsection{The Scale Factors}

To compute the scale factors $s_{\alpha}$, we can start from equation (6). Once we use the proper $P$ and $R$, the power-counting terms all work out correctly, and we are left to consider only the scalar term:

$$
1=s_{\alpha} \prod_{\beta} q_{\beta}^{R_{\alpha \beta}} \prod_{i=1}^{N_{c}} c_{i}^{P_{\alpha i}}
$$

So each $s_{\alpha}$ can be read off by taking the $q_{\beta}$ and $c_{i}$ raised to powers read off by the corresponding elements of $-R_{\alpha \beta}$ and $-P_{\alpha i}$.

For example, in the relativity model, $R_{22}=1, q_{2}$ is one $\mathrm{eV}$ (measured in Joules) $P_{21}=$ -2 , and $c_{1}$ is the speed of light $c$ (measured in $\mathrm{m} / \mathrm{s}$ ). (The other relevant elements of $R$ and $P$ are zero.) So

$$
s_{2}=(e V / \text { Joule })^{-1} c^{2}
$$

\section{$5 \quad$ Examples from the Models}

Here we present the full definitions and computations for each model. Since Amount-ofsubstance and Luminosity never come into non-trivial play, we ignore those dimensions. We are left with 5 dimensions; and we associate $\mathrm{p} 1$ (and $\mathrm{d} 1$ ) with length, p2 (and d2) with mass, p3 (and d3) with time, p4 (and d4) with current, and p5 (and d5) with temperature.

\section{1 rel model}

The constant constraints are

1. $c=1$

The denominating quantities are

1. (dimension corresponding to length is supressed)

2. one $\mathrm{eV}$

3. one second

4. one Amp

5. one Kelvin

Then

$$
\begin{gathered}
C=\left(\begin{array}{rrrrrr}
m & k g & s & A & K & \\
1 & 0 & -1 & 0 & 0 & c_{1}=c
\end{array}\right) \\
Q=\left(\begin{array}{rrrrrrrr}
d 2 & m & k g & s & A & K & & \\
d 3 & 0 & 1 & -2 & 0 & 0 & e V & q_{2}=e V / J \\
d 4 & 0 & 0 & 0 & 0 & 0 & s & q_{3}=1 \\
d 5 & 0 & 0 & 0 & 0 & 1 & K & q_{4}=1 \\
q_{5}=1
\end{array}\right) \\
U=\left(\begin{array}{rrrrrrr}
m & m & k g & s & A & K \\
d 2 & 1 & 0 & -1 & 0 & 0 \\
d 3 & 0 & 0 & 1 & 0 & 0 \\
d 4 & 0 & 0 & 0 & 1 & 0 \\
d 5 & 0 & 0 & 0 & 0 & 1
\end{array}\right)
\end{gathered}
$$




$$
\begin{aligned}
K=U^{-1} & =\left(\begin{array}{rrrrrr} 
& c_{1} & d 2 & d 3 & d 4 & d 5 \\
p 1 & 1 & 0 & 1 & 0 & 0 \\
p 2 & -2 & 1 & 0 & 0 & 0 \\
p 3 & 0 & 0 & 1 & 0 & 0 \\
p 4 & 0 & 0 & 0 & 1 & 0 \\
p 5 & 0 & 0 & 0 & 0 & 1
\end{array}\right) \\
R & =\left(\begin{array}{rrrrr}
p 1 & 0 & 1 & 0 & 0 \\
p 2 & 1 & 0 & 0 & 0 \\
p 3 & 0 & 1 & 0 & 0 \\
p 4 & 0 & 0 & 1 & 0 \\
p 5 & 0 & 0 & 0 & 1
\end{array}\right) \\
& \\
P & =\left(\begin{array}{rrr}
p 1 & 1 \\
p 2 & -2 \\
p 3 & 0 \\
p 4 & 0 \\
p 5 & 0
\end{array}\right)
\end{aligned}
$$

So $\mathrm{d} 1$ is supressed $(\mathrm{d} 1=0), \mathrm{d} 3=\mathrm{p} 1+\mathrm{p} 3$, and the remaining d's are the corresponding p's. (In the original package, we violated this mantra a bit, supressing $\mathrm{d} 3$ and using $\mathrm{d} 1=$ $\mathrm{p} 1+\mathrm{p} 3$ even though seconds (and not meters) is our denominating quantity. This would of course be moot from any external perspective.)

And reading exponents of $-R$ and $-P$ to use as exponents of $q_{\beta}$ and $c_{i}$, we get:

- def_m $=c_{1}^{-P_{11}}=c^{-1}$

- def_kg $=c_{1}^{-P_{21}} q_{2}^{-R_{22}}=c^{2 *}(e V / J)^{-1}$

- def_s $=1$

- def_A $=1$

- def_K $=1$

(Here, terms where $q_{\beta}=1$ are not shown since they do not affect the scale factors regardless of exponent.)

When we show physical constants in this way, we mean measured in std units, for example $c$ is measured in $\mathrm{m} / \mathrm{s}$.

\section{2 hep model}

Now we add the constraint that $k$ (which in std is measured in $\mathrm{J} / \mathrm{K}$ ) is one, change over to $\mathrm{GeV}$ and ns, and also demoninate charge in units of $e^{+}$.

The constant constraints are
1. $c=1$
2. $k=1$

The denominating quantities are 
1. (dimension corresponding to length is supressed)

2. one $\mathrm{GeV}$

3. one nanosecond

4. one electron-charge (= eplus/Coulomb Amp-Seconds)

5. (dimension corresponding to temperature is supressed)

(In this and subsequent models, we also denominate Amount-of-substance in molecules; this was intended but not done in the original package. For the purpose of this note we are ignoring that $\mathrm{p} 6$ dimension.)

$$
C=\left(\begin{array}{rrrrrr}
m & k g & s & A & K & \\
1 & 0 & -1 & 0 & 0 & c_{1}=c \\
2 & 1 & -2 & 0 & -1 & c_{5}=k
\end{array}\right)
$$

(We call the second contraint $c_{5}$ so that there will be a nice coverage of indices: For any $i$ there will be either a non-suppressed $d_{i}$ or a $c_{i}$.)

$$
\begin{aligned}
& Q=\left(\begin{array}{rrrrrrrl} 
& m & k g & s & A & K & & \\
d 2 & 2 & 1 & -2 & 0 & 0 & G e V & q_{2}=G e V / J \\
d 3 & 0 & 0 & 1 & 0 & 0 & n s & q_{3}=n s / s=10^{-9} \\
d 4 & 0 & 0 & 1 & 1 & 0 & e^{+} & q_{4}=e^{+} / \text {Coulomb }
\end{array}\right) \\
& U=\left(\begin{array}{rrrrrr} 
& m & k g & s & A & K \\
& 1 & 0 & -1 & 0 & 0 \\
d 2 & 2 & 1 & -2 & 0 & 0 \\
d 3 & 0 & 0 & 1 & 0 & 0 \\
d 4 & 0 & 0 & 1 & 1 & 0 \\
& 2 & 1 & -2 & 0 & -1
\end{array}\right) \\
& K=U^{-1}=\left(\begin{array}{rrrrrr} 
& c_{1} & d 2 & d 3 & d 4 & c_{5} \\
p 1 & 1 & 0 & 1 & 0 & 0 \\
p 2 & -2 & 1 & 0 & 0 & 0 \\
p 3 & 0 & 0 & 1 & 0 & 0 \\
p 4 & 0 & 0 & -1 & 1 & 0 \\
p 5 & 0 & 1 & 0 & 0 & -1
\end{array}\right) \\
& R=\left(\begin{array}{rrrr} 
& d 2 & d 3 & d 4 \\
p 1 & 0 & 1 & 0 \\
p 2 & 1 & 0 & 0 \\
p 3 & 0 & 1 & 0 \\
p 4 & 0 & -1 & 1 \\
p 5 & 1 & 0 & 0
\end{array}\right) \\
& P=\left(\begin{array}{rrr} 
& c_{1} & c_{5} \\
p 1 & 1 & 0 \\
p 2 & -2 & 0 \\
p 3 & 0 & 0 \\
p 4 & 0 & 0 \\
p 5 & 0 & -1
\end{array}\right)
\end{aligned}
$$


So $\mathrm{d} 1$ and $\mathrm{d} 5$ are supressed $(\mathrm{d} 1=\mathrm{d} 5=0), \mathrm{d} 3=\mathrm{p} 1+\mathrm{p} 3-\mathrm{p} 4$, and $\mathrm{d} 2=\mathrm{p} 2+\mathrm{p} 5$. (In the original package, we again violated this mantra a bit, supressing the $\mathrm{d} 3$ dimension even though we denominate in seconds.)

And reading exponents of $-R$ and $-P$ to use as exponents of $q_{\beta}$ and $c_{i}$, we get:

- def_m $=c_{1}^{-P_{11}}=c^{-1}$

- def_kg $=c_{1}^{-P_{21}} q_{2}^{-R_{22}}=c^{2 *}(\mathrm{GeV} / J)^{-1}$

- $\operatorname{def} \_s_{s}=q_{3}^{-R_{33}}=(n s / s)^{-1}=10^{9}$

- def_A $=q_{3}^{-R_{43}} q_{4}^{-R_{44}}=(n s / s)^{+1}\left(e^{+} / \text {Coulomb }\right)^{-1}=10^{-9} *\left(e^{+} / \text {Coulomb }\right)^{-1}$

- def_K $=c_{5}^{-P_{55}} q_{2}^{-R_{52}}=k^{+1 *}(\mathrm{GeV} / J)^{-1}$

Note that in this model, even though we fix the charge on the positron to be +1 , we do that by choosing how we denominate charge, not by using a phsyical constant constraint to supress the current dimension. Thus dimension checking still goes on for quantities having a net power of current (or charge).

\section{3 qtm model}

Now we add the constraints that $\hbar=1$, and $\varepsilon_{0}=1$. We continue to denominate in $\mathrm{GeV}$, but the because of the added constraints we no longer use ns to denominate time, nor $e^{+}$to denominate charge. (With $\varepsilon_{0}$ set to unity, the charge on the electron is fixed by the square root of the fine structure constant.)

The constant constraints are

1. $c=1$

2. $\hbar=1$

3. $\varepsilon_{0}=1$ (This is measured in Farads/meter)

4. $k=1$

The denominating quantities are

1. (dimension corresponding to length is supressed)

2. one $\mathrm{GeV}$

3. (dimension corresponding to time is supressed)

4. (dimension corresponding to current is supressed)

5. (dimension corresponding to temperature is supressed)

$$
\begin{aligned}
& C=\left(\begin{array}{crrrrr}
m & k g & s & A & K & \\
1 & 0 & -1 & 0 & 0 & c_{1}=c \\
2 & 1 & -1 & 0 & 0 & c_{3}=\hbar \\
-3 & -1 & 4 & 2 & 0 & c_{4}=\varepsilon_{0} \\
2 & 1 & -2 & 0 & -1 & c_{5}=k
\end{array}\right) \\
& Q=\left(\begin{array}{rrrrrrrl} 
& m & k g & s & A & K & & \\
d 2 & 2 & 1 & -2 & 0 & 0 & G e V \quad q_{2}=G e V / J
\end{array}\right)
\end{aligned}
$$




$$
\begin{array}{r}
U=\left(\begin{array}{rrrrr}
m & k g & s & A & K \\
1 & 0 & -1 & 0 & 0 \\
d 2 & 1 & -2 & 0 & 0 \\
2 & 1 & -1 & 0 & 0 \\
2 & -1 & 4 & 2 & 0 \\
2 & 1 & -2 & 0 & -1
\end{array}\right) \\
K=U^{-1}=\left(\begin{array}{rrrrrr}
p 1 & c_{1} & d 2 & c_{3} & c_{4} & c_{5} \\
p 2 & -2 & -1 & 1 & 0 & 0 \\
p 3 & 0 & -1 & 0 & 0 & 0 \\
p 4 & .5 & 1 & -.5 & .5 & 0 \\
p 5 & 0 & 1 & 0 & 0 & -1
\end{array}\right)
\end{array}
$$

Notice that tor the first time, fractional powers are creeping in. This is nothing to worry about; but absent a solid formalism it would make the computation of scale factors quite complicated.

$$
\begin{gathered}
R=\left(\begin{array}{rr}
p 1 & d 2 \\
p 2 & 1 \\
p 3 & -1 \\
p 4 & 1 \\
p 5 & 1
\end{array}\right) \\
P=\left(\begin{array}{rrrrr} 
& c_{1} & c_{3} & c_{4} & c_{5} \\
p 1 & 1 & 1 & 0 & 0 \\
p 2 & -2 & 0 & 0 & 0 \\
p 3 & 0 & 1 & 0 & 0 \\
p 4 & .5 & -.5 & .5 & 0 \\
p 5 & 0 & 0 & 0 & -1
\end{array}\right)
\end{gathered}
$$

So $\mathrm{d} 1, \mathrm{~d} 3, \mathrm{~d} 4$ and $\mathrm{d} 5$ are supressed $(\mathrm{d} 1=\mathrm{d} 3=\mathrm{d} 4=\mathrm{d} 5=0)$, and $\mathrm{d} 2=\mathrm{p} 2+\mathrm{p} 4+\mathrm{p} 5-\mathrm{p} 1-\mathrm{p} 3$.

And reading exponents of $-R$ and $-P$ to use as exponents of $q_{\beta}$ and $c_{i}$, we get:

- def_m $=c_{1}^{-P_{11}} c_{3}^{-P_{13}} q_{2}^{-R_{12}}=c^{-1} \hbar^{-1} *(G e V / J)^{+1}$

- def_kg $=c_{1}^{-P_{21}} q_{2}^{-R_{22}}=c^{2 *}(\mathrm{GeV} / J)^{-1}$

- def_s $=c_{3}^{-P_{33}} q_{2}^{-R_{32}}=\hbar^{-1} *(G e V / J)^{+1}$

- def_A $=c_{1}^{-P_{41}} c_{3}^{-P_{43}} c_{4}^{-P_{44}} q_{2}^{-R_{42}}=\frac{1}{\sqrt{c}} * \sqrt{\hbar} * \frac{1}{\sqrt{\varepsilon_{0}}} *(G e V / J)^{-1}$

- def_K $=c_{5}^{-P_{55}} q_{2}^{-R_{52}}=k^{+1} *(G e V / J)^{-1}$

\section{4 nat model}

Finally we add one more constraint: The gravitational constant $G=1$. Now the last of the dimensinal quantities goes away, and we have no freedom concerning how to denominate quantities. We work in units of the Planck mass, Planck length, Planck time, and so forth.

The constant constraints are 
1. $c=1$

2. $G=1$ (This is measured in cubic meters $/ \mathrm{kg}$ per second squared)

3. $\hbar=1$

4. $\varepsilon_{0}=1$ (This is measured in Farads/meter)

5. $k=1$

There are no denominating quantities.

1. (dimension corresponding to length is supressed)

2. (dimension corresponding to mass is supressed)

3. (dimension corresponding to time is supressed)

4. (dimension corresponding to current is supressed)

5. (dimension corresponding to temperature is supressed)

$$
\begin{array}{r}
C=U=\left(\begin{array}{rrrrrr}
m & k g & s & A & K & \\
1 & 0 & -1 & 0 & 0 & c_{1}=c \\
3 & -1 & -2 & 0 & 0 & c_{2}=G \\
2 & 1 & -1 & 0 & 0 & c_{3}=\hbar \\
-3 & -1 & 4 & 2 & 0 & c_{4}=\varepsilon_{0} \\
2 & 1 & -2 & 0 & -1 & c_{5}=k
\end{array}\right) \\
P=K=U^{-1}=\left(\begin{array}{rrrrrrr}
p 1 & -1.5 & 0.5 & 0.5 & 0.0 & 0.0 \\
p 2 & 0.5 & -0.5 & 0.5 & 0.0 & 0.0 \\
p 3 & -2.5 & 0.5 & 0.5 & 0.0 & 0.0 \\
p 4 & 3.0 & -0.5 & 0.0 & 0.5 & 0.0 \\
p 5 & 2.5 & -0.5 & 0.5 & 0.0 & -1.0
\end{array}\right)
\end{array}
$$

$\mathrm{d} 1, \mathrm{~d} 2, \mathrm{~d} 3, \mathrm{~d} 4$ and $\mathrm{d} 5$ are all supressed $(\mathrm{d} 1=\mathrm{d} 2=\mathrm{d} 3=\mathrm{d} 4=\mathrm{d} 5=0)$.

And reading exponents of $-P$ to use as exponents of $c_{i}$, we get:

- def_m $=c_{1}^{-P_{11}} c_{2}^{-P_{12}} c_{3}^{-P_{13}}=\sqrt{c^{3} G^{-1} \hbar^{-1}}$

- def_kg $=c_{1}^{-P_{21}} c_{2}^{-P_{22}} c_{3}^{-P_{23}}=\sqrt{c^{-1} G^{+1} \hbar^{-1}}$

- def_s $_{-}=c_{1}^{-P_{31}} c_{2}^{-P_{32}} c_{3}^{-P_{33}}=\sqrt{c^{5} G^{-1} \hbar^{-1}}$

- def_A $=c_{1}^{-P_{41}} c_{2}^{-P_{42}} c_{4}^{-P_{44}}=c^{-3} \sqrt{G^{+1} \varepsilon_{0}^{-1}}$

- def_K $=c_{1}^{-P_{51}} c_{2}^{-P_{52}} c_{3}^{-P_{53}} c_{5}^{-P_{55}}=k^{+1} \sqrt{c^{-5} G^{+1} \hbar^{-1}}$ 


\section{Where is this Computed or Embedded in SIunits}

(Since this is going to change, we will make this very brief for now.)

The mapping is expressed as a set of enums expressing $\{\mathrm{d} 1 \ldots \mathrm{d} 7\}$ in terms of linear combinations of $\{\mathrm{p} 1 \ldots \mathrm{p} 7\}$.

The scale factors are computed in calibrate.cc. For each model, the results of the matrix inversion and subsequent steps to get the scale factors in terms of std model values of constraint constants, are coded into the computation of the various scales such as def_m. For example, in the method $\operatorname{rel}()$, we have

def_kg $=c 2$. measuredIn $(\mathrm{m} 2 / \mathrm{s} 2) * \mathrm{~J} . \operatorname{measured} \operatorname{In}(\mathrm{eV})$;

which reflects equation (11). 